Quantum Optics (to be published): LA-UR-93-2804

\title{
RYDBERG WAVE PACKETS ARE SQUEEZED STATES
}

\author{
Michael Martin Nieto ${ }^{a}$ \\ Theoretical Division, Los Alamos National Laboratory \\ University of California \\ Los Alamos, New Mexico 87545, U.S.A.
}

\begin{abstract}
We point out that Rydberg wave packets (and similar "coherent" molecular packets) are, in general, squeezed states, rather than the more elementary coherent states. This observation allows a more intuitive understanding of their properties; e.g., their revivals.
\end{abstract}

PACS: 03.65.-w, 02.20.+b, 42.50.-p

Over the past decade, a very interesting phenomenon has been observed experimentally and discussed theoretically [1, 2, 3, 4], Rydberg wave packets. A short-pulsed laser beam is used to excite a high- $\langle n\rangle$ "Rydberg wave packet." This packet often has a significant overlap with a small number of eigenstates, it exhibits classical motion for a short period, it disperses, and then undergoes periodic revivals. As such, these systems are often referred as being in a coherent state. In this short letter we point out that, actually, these packets are composed of generalized squeezed states, as are some other coherent quantum systems, such as laser excited molecules [5].

To set the stage, let us quickly review some of the important properties of the squeezed states of the harmonic oscillator [6, 7]. From the wave-function point of view, these states minimize the $x-p$ uncertainty relation at $t=0$. They are Gaussians, displaced from the origin, whose width is not that of the ground state:

$$
\psi_{s s}(x)=\left[\frac{\omega}{\pi s^{2}}\right]^{1 / 4} \exp \left[-\frac{\omega\left(x-x_{0}\right)^{2}}{2 s^{2}}+i p_{0} x\right]
$$

where $\hbar=m=1$. If $s=1$, the Gaussians have the width of the ground state of the harmonic oscillator. Then they are the special case of coherent states.

Both coherent states and squeezed states follow the classical motion in that

$$
\langle x\rangle_{c s}=\langle x\rangle_{s s}=x_{c l}=x_{0} \cos (\omega t)+\frac{p_{0}}{\omega} \sin (\omega t) .
$$


However, their uncertainty products vary with time as

$$
\begin{gathered}
{[\Delta x(t)]^{2}=\frac{1}{2 \omega}\left[s^{2} \cos ^{2} \omega t+\frac{1}{s^{2}} \sin ^{2} \omega t\right]} \\
{[\Delta p(t)]^{2}=\frac{\omega}{2}\left[\frac{1}{s^{2}} \cos ^{2} \omega t+s^{2} \sin ^{2} \omega t\right],} \\
{[\Delta x(t)]^{2}[\Delta p(t)]^{2}=\frac{1}{4}\left[1+\frac{1}{4}\left(s^{2}-\frac{1}{s^{2}}\right) \sin ^{2}[2 \omega t]\right] .}
\end{gathered}
$$

Thus, although the $s=1$ coherent states have an unvarying uncertainty product and maintain their shapes, the squeezed states do not. As Eq. (3) shows, four times every classical period a squeezed state will return to the coherent-state minimumuncertainty value for position, $1 / 2 \omega$; specifically when

$$
\sin ^{2} \omega t=\left(\frac{s^{2}}{1+s^{2}}\right)
$$

For larger or smaller values of $t$, the uncertainty value will be different. A similar statement holds for momentum.

These are a type of "revival," which indeed is a specialized flowering of a general phenomenon. It has long been known that in a system with equally-spaced eigenenergies, any wavepacket will reconstruct itself every classical period of oscillation [8]. This is simply because the decomposition of the wave function into eigenstates,

$$
\Psi(x, t)=\sum_{n=0}^{\infty} c_{n} \exp \left[-i \omega_{0} t\left(n+n_{0}\right)\right] \psi_{n}(x)
$$

means the wave function will return to its original shape after every period $t=2 \pi / \omega_{0}$. Gaussians, in a harmonic oscillator potential, do even better.

Another property of squeezed states vs. coherent states is that they can be constructed so as to significantly overlap fewer eigenstates (sub Poisson number distribution) or more eigenstates (super Poisson distribution) than a coherent state would (Poisson distribution). For a squeezed state (now $\omega=1$ )

$$
\begin{gathered}
\langle N\rangle=\alpha_{1}^{2}+\alpha_{2}^{2}+\left[\frac{\left(s^{2}-1\right)^{2}}{4 s^{2}}\right] \\
(\Delta N)^{2}=\alpha_{1}^{2} s^{2}+\frac{\alpha_{2}^{2}}{s^{2}}+\left[\frac{\left(s^{2}+1\right)^{2}}{2 s^{2}}\right]\left[\frac{\left(s^{2}-1\right)^{2}}{4 s^{2}}\right],
\end{gathered}
$$


where $\alpha=\alpha_{1}+i \alpha_{2}=x_{0} / \sqrt{2}+i p_{0} / \sqrt{2}$ is the usual coherent state parameter and $s=1$ is the limit to a coherent state. Further, for large squeezing, oscillations occur in the probability distribution as a function of $n$ [9].

Consider, in the above, the cases where $\alpha_{1}^{2}=0, \alpha_{2}^{2} \sim 64$, and $s^{2}=4$ or $1 / 4$, respectively. Then $(\Delta N) \sim\langle N\rangle^{1 / 2} / s \sim 4$ or 16, respectively, instead of 8 , as would be the case for a coherent state. Therefore, such states would exhibit "squeezing" in their evolution and revivals.

One can use Gaussians as approximate states to describe physical situations. However, for more general systems, more general coherent and squeezed states are called for as a matter of principle. Their advantage is to better take into account the different shapes of the various potentials, especially the centripetal barriers of angular momentum. Because of these different shapes, packets will evolve differently, but one still wants to maximize the coherence properties of the wave packet.

There exists a method to obtain coherent and squeezed states for general systems that was inspired by Schrödinger's original discovery of coherent states [10]. Schrödinger was interested in finding states which follow the classical motion and do not charge their shapes with time. His expressed belief that the same could be done for the Kepler problem led to disagreement between he and Heisenberg [11]. The controversy resulted in Heisenberg's discovery of the uncertainty relation [12] and Schrödinger's generalization of it [13].

In this method one starts with the classical problem and transform it to the "natural classical variables," $X_{c}$ and $P_{c}$, which vary as the sin and the cos of the classical $\omega t$ (or $\theta(t)$, for spherical systems). The Hamiltonian then is of the form $P_{c}^{2}+X_{c}^{2}$. Now take these natural classical variables and transform them into "natural quantum operators." Since these are quantum operators, they have a commutation relation and uncertainty relation:

$$
[X, P]=i G, \quad(\Delta X)^{2}(\Delta P)^{2} \geq \frac{1}{4}\langle G\rangle^{2}
$$

The states that minimize the uncertainty relation above are the solutions to

$$
Y \psi_{s s} \equiv\left(X+\frac{i\langle G\rangle}{2(\Delta P)^{2}} P\right) \psi_{s s}=\left(\langle X\rangle+\frac{i\langle G\rangle}{2(\Delta P)^{2}}\langle P\rangle\right) \psi_{s s}
$$

Of the four parameters $\langle X\rangle,\langle P\rangle,\left\langle P^{2}\right\rangle$, and $\langle G\rangle=\Delta X \Delta P$, only three are independent because the uncertainty relation is satisfied. Therefore,

$$
(X+i B P) \psi_{s s}=C \psi_{s s}, \quad B=\frac{\Delta X}{\Delta P}, \quad C=\langle X\rangle+i B\langle P\rangle
$$

Here $B$ is real and $C$ is complex. These states, $\psi_{s s}(B, C)$, are the minimum-uncertainty or squeezed states for general potentials [14, 15]. $B$ can be adjusted to $B_{0}$ so that the 
ground eigenstate of the potential is a member of the set. Then $\psi_{s s}\left(B=B_{0}, C\right)=$ $\psi_{c s}\left(B_{0}, C\right)$, are the coherent states for general potentials. (Recently this method was connected to a ladder-operator method for obtaining generalized squeezed states [16].)

The natural quantum operators follow the quantum equations of motion. Therefore, the coherent and squeezed state expectation values of these operators follow the classical equations of motion. Intuitively, one can easily understand that if a harmonic oscillator potential is slightly deformed to another potential, the appropriate coherent and squeezed states will still retain their basic properties of classical motion, revivals, etc. What happens here is the opposite. A general potential is classically transformed to a harmonic oscillator-like potential by the appropriate change of variables. This allows the quantum-mechanical coherent and squeezed states to be obtained for this pseudo-harmonic oscillator in terms of the physical operators.

When applied to the Coulomb problem, the natural quantum operators are [17]

$$
\begin{aligned}
& X=\left(\frac{1}{\rho}-\frac{1}{2 l(l+1)}\right), \\
& P=p_{r}=\frac{1}{i}\left(\frac{1}{\rho}+\frac{d}{d \rho}\right),
\end{aligned}
$$

where $\rho$ is the dimensionless radius and $p_{r}$ is the dimensionless radial momentum. These operators obey the quantum analogues to the classical equations of motion:

$$
\begin{gathered}
\dot{X}=\frac{1}{i}[X, H]=-\frac{1}{2}\left\{\frac{1}{\rho^{2}}, P\right\} \\
\dot{P}=\frac{1}{i}[P, H]=\frac{l(l+1)}{\rho^{2}} X .
\end{gathered}
$$

The squeezed states obtained from these operators are

$$
\begin{gathered}
\psi_{s s}=[2 B\langle 1 / \rho\rangle]^{B+1 / 2} \Gamma(2 B+1)^{-1 / 2} \rho^{B-1} \exp [-C \rho], \\
B=\frac{\left\langle 1 / \rho^{2}\right\rangle}{2[\Delta(1 / \rho)]^{2}}, \quad C=B\langle 1 / \rho\rangle-i\left\langle p_{r}\right\rangle \equiv u+i v,
\end{gathered}
$$

When $B=l+1$, these states are the coherent states. The Coulomb eigenstates are

$$
\psi_{n l}=\left[\frac{\Gamma(n-l)}{2 n^{4} \Gamma(n+l+1)}\right]^{1 / 2} \exp \left[-\rho_{n} / 2\right] \rho_{n}^{l} L_{n-l-1}^{(2 l+1)}\left(\rho_{n}\right),
$$


where $\rho_{n}=\rho / n$ and the $\mathrm{L}$ are the associated Laguerre polynomials. Therefore, the Coulomb ground state $(n=l+1)$ is a coherent state. (The decomposition of the coherent and squeezed states into number states is straight forward since

$\int_{0}^{\infty} d x x^{n} \exp [-b x] L_{k}^{(\alpha)}(x)$ is a standard integral.) Numerical studies of the coherent [18] and squeezed [19] states have both been done, and they show the appropriate characteristics, including revivals. For example, Fig. 1, taken from Ref. [18], shows the early time-evolution of a minimum-uncertainty Coulomb coherent state.

The Rydberg wave packets which have been discussed in the literature are usually taken as phenomenological Gaussians. For large- $n$, these are a good approximation to the appropriate squeezed states because, as has been noted [1, 2, 3, 4], the eigenenergies are approximately equally spaced. In particular, one can compare the squeezed Gaussian ( $x$ becoming the radial distance $\rho$ ) and the Coulomb squeezed state above. With $p_{0}=0$ and $C$ real, one can match the highest point of the wave packets if $x_{0}=(B-1) / C$. Then the ratio of the zeroth-order to the second-order Taylor series about $x_{0}$ can be matched if $s^{2}=(B-1) / C^{2}$.

Of course, there have been discussions of other "coherent states" for the Coulomb problem [20]. An advantage of our point of view is that the states are obtained from a general method applicable to arbitrary potentials, and the connection to the associated squeezed states is also explicit and general. In particular, it should be noted that similar comments about squeezing can be made about other "coherent" quantum systems, such as molecules excited by short laser pulses [5].

A final comment on what can be meant by "coherent" and "squeezed" states. For the harmonic oscillator, the concepts "coherent" and "squeezed" are well-defined and understood by all. But supposing one took a coherent state, and distorted it's wavepacket probability form by $1 \%$ with wiggles at the edges of the packet. Would that packet still "cohere?" Of course it would, and one would not notice much difference.

This demonstrates a distinction which must be kept in mind. One set of definitions of coherent and squeezed states is composed of precise mathematical definitions, be they from the i) minimum-uncertainty, ii) ladder-operator, or iii) displacementoperator points of view. They are based on mathematical and/or physical criterion.

On the other hand, there are phenomenological definitions based on approximate criteria. Intuitively, these criteria are that the center of the wave packet follows the classical motion as well as possible, and that the shape of the wave packet remains the same, or returns to its original shape periodically, as well as possible. For historical or simplicical reasons, these approximate definitions are very often simply taken to yield Gaussians. These Gaussians may indeed be good approximations to more precise definitions, as in this large- $n$ Rydberg case. Further, since they are packets and not spread-out over a large area, they will "cohere" and/or "squeeze" to some, perhaps very good, approximation in the system under study.

As long as one keeps these separate uses of the words "cohere," "coherent," and "squeezed" clear, then the important thing, the physics, will not become confused.

I wish to thank Wolfgang Schleich and Carlos Stroud, Jr., for helpful comments. Also note that related conclusions have been obtained by Bluhm and Kostelecký [19], based on the supersymmetry-inspired quantum defect-theory [21]. 
Email: ${ }^{a}$ mmn@pion.lanl.gov

\section{References}

[1] Parker J and Stroud, Jr. C R 1986 Phys. Rev. Lett. 56 716-719

Yeazell J A, Mallalieu M, and Stroud, Jr. C R 1990 Phys. Rev. Lett. $642007-$ 2010

Yeazell J A and Stroud, Jr. C R 1991 Phys. Rev. A 43 5153-5156

[2] ten Wolde A, Noordam L D, Lagendijk A, and van Linden van den Heuvell H B 1988 Phys. Rev. Lett 61 2099-2101

ten Wolde A, Noordam L D, Lagendijk A, and van Linden van den Heuvell H B 1989 Phys. Rev. A 40 485-488

[3] Averbukh I Sh and Perelman N F 1989 Phys. Lett. A 139 449-453

[4] Alber G and Zoller P 1991 Phys. Rep. 199 231-280, and references therein

[5] Garraway B, Stenholm S, and Kalle-Antti S 1993 Physics World 6 No. 4 46-51, and references therein

[6] Schumaker B L 1986 Phys. Rep. 135 317-408, and references therein

[7] For a pedagogical review, see

Nieto M M 1986 Frontiers of Nonequilibrium Statistical Physics ed G. T. Moore and M. O. Scully (Plenum, New York) p 287.

[8] Kennard E H 1927 Zeit. Phys. 44 326-352

Saxon D S 1964 Elementary Quantum Mechanics, Preliminary Edition (HoldenDay, San Francisco) p 162

[9] Schleich W and Wheeler J A 1987 Nature 326 574-577

Schleich W and Wheeler J A 1987 Opt. Soc. Am. B 4 1715-1722

The $s$ in these authors' notation is $1 / s^{2}$ in our notation.

[10] Schrödinger E 1926 Naturwissenshaften 14 664-666

[11] Steiner F 1988 Physica B 151 323-326

[12] Heisenberg W 1927 Zeit. Phys. 43 172-198

[13] Schrödinger E 1930 Sitzungsber. Preuss. Akad. Wiss. Berlin, Math. Phys. Kl. 19 296-303

[14] Nieto M M and Simmons, Jr. L M 1978 Phys. Rev. Lett. 41 207-210

Nieto M M and Simmons, Jr. L M 1979 Phys. Rev. D 20 1321-1331, the first of a series concluding with

Nieto M M, Simmons, Jr. L M, and Gutschick V P 1981 Phys. Rev. D 23 927-933 
[15] As noted in Ref. [7], there were many discoveries of what are now called squeezed states, with many names attached to these states. The term "squeezed" was invented by

Hollenhorst J N 1979 Phys. Rev. D 19 1669-1679 in the context of gravitational wave detection. Within a few years, this terminology came to dominate.

[16] Nieto M M and Truax D R 1993 Phys. Rev. Lett. (to b published).

[17] Nieto M M 1980 Phys. Rev. D 22 391-402

[18] Gutschick V P and Nieto M M 1980 Phys. Rev. D 22 403-418

[19] Bluhm R and Kostelecký V A 1993 Indiana University Preprints IUHET-255 and IUHET-256

[20] Brown L S 1973 Am. J. Phys. 41 525-530 (1973)

Mostowski J 1977 Lett. Math. Phys. 2 1-5

Bhaumik D, Dutta-Roy B, and Ghosh G 1986 J. Phys. A 19, 1355-1364

Gerry C C 1986 Phys. Rev. A 33 6-11

Nauenberg M 1989 Phys. Rev. A 40 1133-1136

[21] Kostelecký V A and Nieto M M 1985 Phys Rev. A 32 3243-3246

Kostelecký V A, Nieto M M, and Truax D R 1988 Phys. Rev. A 38 4413-4418

Bluhm R and Kostelecký V A 1993 Phys Rev. A 47 794-808

Figure 1. The time-evolution of a minimum-uncertainty Coulomb coherent state. $l=150$, where $B=l+1$. $C$ (comnplex) is adjusted so that the particle starts half-way in time between the classical turning points. The energy, $\langle H\rangle$, is $1 / 5$ the way up to the continuum from the minimum of the potential. The number at the top of the frames indicates the periods of revolution. 
This figure "fig1-1.png" is available in "png" format from: http://arxiv.org/ps/hep-ph/9310343v1 